\title{
ANÁLISIS PSICOMÉTRICO DE LA ESCALA DE CANSANCIO EMOCIONAL EN ESTUDIANTES DE UNA UNIVERSIDAD PRIVADA
}

\section{PSYCHOMETRIC ANALYSIS OF THE EMOTIONAL EXHAUSTION SCALE OF PRIVATE UNIVERSITY STUDENTS}

\author{
Sergio Alexis Dominguez Lara* \\ Universidad Inca Garcilaso de la Vega, Perú
}

\section{RESUMEN}

El objetivo del estudio instrumental fue analizar las propiedades psicométricas de la Escala de Cansancio Emocional (ECE) en una muestra de 233 estudiantes de Psicología de una universidad privada con edades comprendidas entre 16 y 44 años (M=20.9). El análisis factorial realizado revela que la ECE presenta una estructura unidimensional (factor que explica 43.361\% de la varianza) y se encontró una correlación positiva y significativa con Ansiedad y Depresión, y correlación negativa y significativa con Autoeficacia Académica. Por otro lado, con relación a la confiabilidad, el coeficiente alfa ordinal es adecuado (.853). Se concluye que la ECE cuenta con propiedades psicométricas adecuadas para seguir con estudios de validación, apoyando así su uso como instrumento de evaluación del cansancio emocional.

Palabras clave: cansancio emocional, confiabilidad, validez, estudiantes universitarios.

\section{ABSTRACT}

The aim of this instrumental study was to analyze the psychometric properties of the Emotional Exhaustion Scale (EES) in a sample of 233 private university students of psychology with ages between of 16 and 44 years old ( $M=20.9)$. The factor analysis reveals that the EES presents a one-dimensional structure (a factor that explains $43.361 \%$ of the total variance) and there was a significant and positive correlation between anxiety and depression and a significant and negative correlation with Academic Self-Efficacy. Furthermore, regarding reliability, the ordinal alpha is adequate (.853). To conclude, the EAPESA has suitable psychometric properties to continue validation studies, which reaffirms its use as an instrument to evaluate emotional exhaustion.

Keywords: emotional exhaustion, reliability, validity, college students. 
El ingreso a la universidad supone, en muchos aspectos, una exigencia mayor desde el punto de vista académico, donde el estudiante se ve en la necesidad de implementar estrategias que faciliten la comprensión y adquisición significativa de contenidos curriculares.

Durante la permanencia del estudiante en la universidad habrá exigencias que necesitan ser afrontadas con éxito para poder lograr los objetivos que se plantea el educando. En ese proceso de exigencia y afrontamiento, tanto los aspectos cognitivos como motivacionales juegan un papel clave en la organización de la conducta académica que llevan a cabo los estudiantes para realizar las actividades que les demanda la vida universitaria (Alonso, 1995; Mas \& Medinas, 2007). De este modo, para que el estudiante tenga un desempeño exitoso, es necesariotener las habilidades que exigen dichas demandas, pero además tener la certeza de que va a lograr buenos resultados a partir de su accionar (Sánchez, Castañeiras \& Posada, 2011).

En este sentido, el estudiante está involucrado en situaciones que le generan estrés, tales como su desempeño académico, su adaptación a las nuevas exigencias y la preocupación por su futuro, así como los horarios, tareas, evaluaciones, etc. (Peñacoba \& Moreno, 1999); que de nos ser afrontadas de forma adecuada, traería como consecuencia el burnout ("síndrome del quemado"), es decir, el cansancio emocional que lleva a la pérdida de motivación y que suele progresar hasta sentimientos de inadecuación y fracaso, lo que explica el deterioro físico y mental (Maslach, Schaufeli \& Leiter, 2001). La primera etapa de este síndrome es el cansancio emocional, que es la respuesta más destacada ante una situación estresante (Gonzáles \& Landero, 2007b), y provoca en el individuo distanciamiento emocional y cognitivo respecto a su propio trabajo (Maslach et al., 2001). Esta dimensión es la que predomina en los estudiantes, ya que las otras dimensiones denominadas despersonalización y falta de realización personal del planteamiento de Maslach et al. (2001) no se observan significativamente en ellos (Martínez- Martínez \& Marques Pinto, 2005).

El cansancio emocional es un aspecto sumamente importante para tener en cuenta. Se ha encontrado que está directamente relacionado a la dimensión neuroticismo y problemas de salud psicológica, e inversamente relacionado al autoconcepto y autoestima (Ramos, Manga \& Morán, 2005; Gil-Monte, 2005; Gonzáles \& Landero, 2007b). La baja autoeficacia también es un componente importante en el desarrollo del burnout académico (Bresó \& Salanova, 2005), estableciéndose como predictor de este. Es decir, mientras mayor es el cansancio emocional, se incrementan las emociones negativas y eto repercute de manera negativa en la autoestima y el autoconcepto. Asimismo, los estudiantes con menor satisfacción con los estudios presentan mayor cansancio emocional (Ramos et al., 2005). Del mismo modo, se encuentra directamente relacionado con la ansiedad y depresión (Aluja, 1997; Neveu, 2007).

Es así que la fase inicial del síndrome, el cansancio emocional, afecta negativamente las expectativas de éxito y la madurez profesional de los estudiantes universitarios (Gonzáles \& Landero, 2007b). No obstante, para obtener resultados verosímiles, deben emplearse instrumentos de evaluación válidos y confiables, de tal manera que la medida de dicho constructo se haga sobre una base sólida que garantice su adecuada interpretación.

En cuanto a los instrumentos para evaluar este aspecto, el uso del Maslash Burnout 
Inventory-MBI (Maslash, 1997) se ha extendido para la evaluación del síndrome de Burnout en estudiantes. Sin embargo, dos de sus tres escalas resultan irrelevantes para su estudio en universitarios (Ramos, et al., 2005; Bonilla, Lira, Balcázar, Enríquez \& Gurrola, 2009). Esto se debe a que la naturaleza, curso y desarrollo del burnout académico son muy diferentes a los del burnout laboral (Caballero, Hederich \& Palacio, 2009), dado que los afectados se encuentran con cargas propias de su labor estudiantil debido a que los contextos son diferentes.

En vista de aquello, Ramos et al. (2005) crearon una escala para la evaluación del cansancio emocional en estudiantes universitarios, la cual ha sido sometida a estudios psicométricos en otros contextos, los cuales se detallan a continuación.

Para la elaboración de la escala, Ramos et al. (2005) contaron con la participación de 402 estudiantes españoles, 130 varones y 272 mujeres entre los 18 y 33 años ( $M=22.65$ años), a quienes administraron la Escala de Cansancio Emocional (ECE), el Inventario NEO reducido de Cinco Factores (NEO-FFI) de Costa y McCrae (1999), la Escala de Autoconcepto Positivo (EAP), la Escala de Autoestima de Rosenberg (1965, en Ramos et al., 2005) y el Cuestionario de Salud General de Goldberg y Williams (1996), además de una medida sencilla de satisfacción con los estudios, la cual constaba de un solo ítem ("Disfruto estudiando porque me gusta lo que estudio") al que la persona respondía en una escala tipo Likert que iba desde raras veces hasta siempre. En cuanto a su confiabilidad, se obtuvieron índices de homogeneidad adecuados, los cuales iban de .43 hasta .61, y un alfa de Cronbach de .83. Por otro lado, con relación a su validez, se empleó un análisis de componentes principales, en el cual se obtuvo una estructura unifactorial que explica el $40 \%$ de la varianza total del instrumento, aunque no se reportan las saturaciones factoriales. La validez convergente dio cuenta de relaciones positivas con neuroticismo y problemas de salud psicológica, y negativas con autoconcepto y autoestima. Por último, en consonancia con el marco teórico de referencia, fueron los estudiantes más insatisfechos los que puntuaron más alto en la escala.

Posteriormente, se estudió la escala en México por Gonzáles y Landero (2007b) contando para ello con 506 estudiantes de Psicología, de los cuales 365 fueron de una universidad pública y 141 de una universidad privada, siendo el $19.2 \%$ varones y el $80.8 \%$ mujeres, de edad promedio 20.71. Se utilizó además de la Escala de Cansancio Emocional, la Escala de Estrés Percibido (Cohen, Kamarak \& Mermelstein, 1983, en Gonzáles \& Landero, 2007a), la Escala de Autoestima de Rosenberg (1989, en Vázquez, Jimenez \& Vázquez, 2004) y la Escala de Ansiedad Social para Adolescentes (La Greca \& López, 1998, en Gonzáles \& Landero, 2007b). Con relación a su confiabilidad, no se reportaron índices de homogeneidad, pero sí un alfa de Cronbach de .90. Por otro lado, con relación a su validez, se empleó un análisis de componentes principales, donde se obtuvo una estructura unifactorial que explica el 52.78\% de la varianza total del instrumento, y cuyas saturaciones factoriales van de .591 hasta .792. Asimismo, mediante el análisis factorial confirmatorio, los datos presentan un ajuste aceptable al modelo unifactorial, aunque no del todo satisfactorio. La validez convergente dio cuenta de relaciones positivas con estrés y ansiedad social, y negativa con autoestima.

También en México, Bonilla et al. (2009) trabajaron la Escala de Cansancio Emocional en una muestra de 154 estudiantes de los últimos años de la carrera de Psicología, de los cuales $73 \%$ eran mujeres y el $27 \%$ varones, de edad media 20.5 años. Con relación a su 
confiabilidad, no se reportaron índices de homogeneidad, pero sí un alfa de Cronbach de .873. Por otro lado, con relación a su validez, se empleó un análisis de componentes principales, en el cual se obtuvo una estructura unifactorial donde se explica el $47.25 \%$ de la varianza total del instrumento, y cuyas saturaciones factoriales van de .50 hasta .805 .

Por último, Fontana (2011) trabajó en Argentina la escala con una muestra de 161 estudiantes de la carrera de Psicología, de los cuales 135 fueron mujeres y 26 varones. La edad media fue de 21.57 años (DE= 2.87). La autora reportó índices de homogeneidad satisfactorios (de .48 a .75), y un alfa de Cronbach de .87. Por otro lado, con relación a su estructura interna, se empleó un análisis factorial de ejes principales, en el cual se obtuvo una estructura unifactorial que explica el $45.56 \%$ de la varianza total del instrumento, cuyas saturaciones factoriales van de .48 hasta .80 .

En Perú no existen instrumentos que tengan indicadores reportados de confiabilidad y validez para evaluar cansancio emocional en estudiantes universitarios. Es por ello que el objetivo del presente estudio fue obtener las primeras evidencias de validez que garanticen su uso en este medio. En este sentido, se examinaron la estructura factorial y la validez convergente de la Escala de Cansancio Emocional en una muestra de estudiantes universitarios de una institución privada.

La utilidad de este estudio versa en las aplicaciones que se le podría dar, ya que conocer estos aspectos traería consecuencias positivas. Desde el punto de vista teórico, podría servir como base para investigaciones posteriores a fin de encontrar un modelo explicativo en la población estudiada; y desde un punto de vista práctico, para la implementación de programas de promoción de estilos de vida saludables o de intervención, según sea el caso, considerando que burnout académico tiene carácter insidioso y puede cursar con formas manifiestas de ansiedad y depresión (Caballero et al., 2009).

\section{MÉTODO}

\section{Diseño}

Según León y Montero (2007) sería un estudio instrumental, destinado a la adaptación y estudio de las propiedades psicométricas de un test.

\section{Participantes}

La muestra de estudio estuvo conformada por 232 estudiantes de Psicología de una universidad privada, 69 varones y 163 mujeres, de edades comprendidas entre $16 \mathrm{y}$ 44 años (Media=20.9). El muestreo utilizado fue de tipo intencional.

\section{Instrumentos}

La Escala de Cansancio Emocional - ECE (Fontana, 2011) consta de 10 ítems (Anexo 1). Los ítems se puntúan de 1 a 5 (de raras veces a siempre), considerando los 12 últimos meses de vida estudiantil. La puntuación obtenida en la ECE oscila entre los 10 y los 50 puntos.

Inventario de Ansiedad Estado-Rasgo (IDARE) de Spielberger y Díaz-Guerrero (2007), en versión de Dominguez, Villegas, Sotelo y Sotelo (2012) estudiada en población universitaria. El inventario comprende escalas separadas de de la ansiedad, tanto Estado (E) como Rasgo (R), donde 20 ítems evalúan la subescala de Estado y 19 ítems la de Rasgo. Presentó una confiabilidad por medio de su consistencia interna de .908 para Ansiedad-Estado y de .874 para AnsiedadRasgo. Asimismo, con relación a la validez factorial, en Ansiedad-Estado se hallaron dos componentes diferenciados, caracterizados por la presencia y ausencia de ansiedad, los cuales explicaron el $48.608 \%$ de la varianza. Por otro 
lado, en la Ansiedad-Rasgo, se encontraron dos componentes que explicaron el $42.110 \%$ de la varianza. Cabe resaltar las evidencias de validez convergente con el Inventario de Depresión Estado-Rasgo. En este estudio se utilizó la versión referida a la Ansiedad- Rasgo y se encontró un alfa de Cronbach de .851.

Inventario de Depresión Estado-Rasgo (IDER) de Spielberger, Agudelo y Buela-Casal (2008), en versión de Dominguez (2011) trabajado en población universitaria. Consta de 20 ítems, diez para Depresión Estado y diez para Depresión Rasgo. Está compuesto por cuatro factores: Distimia estado, que se refiere al grado en el que está presente en el momento de la evaluación un estado de afectividad negativa; Eutimia estado, grado en el que está presente en el momento de la evaluación la afectividad positiva; Distimia rasgo, frecuencia de la presencia de afectividad negativa; y Eutimia rasgo: frecuencia de la presencia de afectividad positiva. Se hallaron indicadores de confiabilidad aceptables a través del método de consistencia interna, así como validez de contenido (V de Aiken superiores a .50) y validez factorial (dos factores que explican el $62.404 \%$ de la varianza en DepresiónEstado, y el 58.455\% en Depresión-Rasgo). En este estudio se utilizó la versión referida a la Depresión-Rasgo y se encontró un alfa de Cronbach de .804 .

\section{Escala de Autoeficacia Percibida} Específica de Situaciones Académicas (EAPESA) de Palenzuela (1983) en versión de Dominguez, Villegas, Yauri, Mattos Ramírez (2012). Consta de 9 ítems con cuatro opciones de respuesta (Nunca, Algunas veces, Bastantes veces y Siempre). Las propiedades psicométricas indican una consistencia interna adecuada (alfa de Cronbach de .89) y una estructura unidimensional, es decir, un solo factor que explica $55.261 \%$ de la varianza total del instrumento. En el presente estudio se encontró un alfa de Cronbach de.91.

\section{Procedimiento}

Se realizó la adaptación de tres ítems de la Escala de Cansancio Emocional, la cual estuvo a cargo de cinco jueces expertos con experiencia en el área, quienes sugirieron modificaciones a los ítems conservando el propósito de éstos, pero adecuando algunos al contexto universitario y la realidad de los estudiantes (Anexo 2).

El inventario se administró como parte de una batería de evaluación psicológica propia de la casa de estudios, por lo cual fue necesario que los evaluados colocaran sus nombres. Los responsables del estudio explicaron las instrucciones que figuran en el protocolo de aplicación de la prueba, y se absolvieron las dudas que las personas evaluadas tuvieron.

El análisis de los datos se llevó a cabo utilizando el programa OPENSTAT (Miller, 2003) para el análisis descriptivo y correlacional, el módulo ViSta-CITA (Ledesma \& Molina, 2009; Young, 2003) en lo que concierne al análisis de ítems; FACTOR (LorenzoSeva \& Ferrando, 2007) para la realización del análisis factorial, y un módulo para el cálculo del alfa ordinal (Dominguez, 2012).

\section{RESULTADOS}

En cuanto al análisis de homogeneidad del test, se examinaron el grado de asociación entre los ítems que conforman la prueba y el test (Elosua, 2003), y se retuvieron aquellos que obtuvieron una correlación ítem-test mayor de .20 (Likert, 1932). Fueron retenidos todos los reactivos en vista que superaron dicho indicador.

Para obtener evidencias de validez, en primer lugar se realizó un análisis factorial exploratorio. Antes de dicho procedimiento, se 
llevóacabounanálisisdeestadísticosdescriptivos y de puntajes extremos, así como la inspección de la matriz de correlaciones policóricas a fin de descartar la existencia de multicolinealidad.

Con relación al análisis descriptivo de los ítems, se constató que estos presentan indicadores de asimetría y curtosis adecuados, dentro del rango +/- 1.5 (Pérez \& Medrano, 2010; Ferrando \& Anguiano-Carrasco, 2010), lo cual se aprecia en la Tabla 1. Del mismo modo, no fue detectada la presencia de puntajes extremos ni de multicolinealidad.

Unavez realizado esto, se exploró si losítems poseían la suficiente correlación para realizar el análisis factorial. En tal sentido, se obtuvo una matriz de correlaciones significativa ( $\mathrm{p}<.01)$; un KMO de .91, valor considerado adecuado (Hair, Anderson, Tatham \& Black, 2005), y un test de esfericidad de Bartlett significativo $(\mathrm{p}<.01)$, lo cual da cuenta de la suficiente intercorrelación de los ítems.

Para la determinación del número de factores se utilizó el análisis paralelo propuesto por Timmerman y Lorenzo-Seva (2011) destinado para variables en escala ordinal, tal como los ítems que componen el instrumento. De acuerdo a dicho método se sugirió la extracción de un solo factor.

Entonces, fue realizado un análisis factorial por mínimos cuadrados no ponderados, sin rotación, en vista que se extraerá un solo factor. El factor obtenido explicó el $43.361 \%$ de la varianza total del instrumento y sus saturaciones factoriales oscilaron entre .517 y .718.

En la tabla 1 presentada a continuación, se aprecian los hallazgos descriptivos, así como los referidos a la estructura factorial del instrumento.

Luego de ello, se obtuvieron evidencias de validez convergente mediante las correlaciones con autoeficacia académica (-.319; $\mathrm{p}<0.001)$, ansiedad-rasgo (.519; p< 0.001) y depresiónrasgo (.373; $\mathrm{p}<0.001)$.

La confiabilidad se estimó mediante el alfa ordinal (Elosua \& Zumbo, 2008; Dominguez, 2012) con lo que se obtuvo un indicador de 853.

Tabla 1

Análisis de la Escala de Cansancio Emocional

\begin{tabular}{cccccccc}
\hline Ítem & Media & $\begin{array}{c}\text { Desv. } \\
\text { Estándar }\end{array}$ & Asimetría & Curtosis & $\begin{array}{c}\text { Correlación } \\
\text { Ítem-Test }\end{array}$ & $\begin{array}{c}\text { Carga } \\
\text { Factorial }\end{array}$ & Comunalidad \\
\hline Ítem 1 & 2.310 & 1.228 & .556 & -.658 & .631 & .668 & .446 \\
Ítem 2 & 2.315 & 1.099 & .449 & -.476 & .503 & .535 & .286 \\
Ítem 3 & 1.810 & .977 & .916 & -.231 & .451 & .525 & .275 \\
Ítem 4 & 2.685 & 1.160 & .102 & -.871 & .520 & .517 & .268 \\
Ítem 5 & 2.134 & 1.052 & .511 & -.528 & .595 & .634 & .402 \\
Ítem 6 & 2.470 & 1.058 & .069 & -.789 & .668 & .665 & .442 \\
Ítem 7 & 2.159 & .976 & .374 & -.665 & .622 & .645 & .416 \\
Ítem 8 & 2.591 & 1.026 & .140 & -.545 & .591 & .579 & .335 \\
Ítem 9 & 2.608 & 1.241 & .207 & -.968 & .734 & .718 & .515 \\
Ítem 10 & 2.496 & 1.063 & .184 & -.565 & .578 & .582 & .339 \\
Total & 23.578 & 7.441 & & & & & \\
\hline
\end{tabular}




\section{DISCUSIÓN}

Con relación al índice de homogeneidad, todos los reactivos fueron aceptados, en vista de que superaron el límite mínimo que es .20 (Likert, 1932). Estos hallazgos concuerdan tanto con Fontana (2011) y Ramos et al. (2005), quienes obtuvieron índices de homogeneidad de 0.48 a 0.75 y de 0.43 hasta 0.61 , respectivamente. En las dos investigaciones citadas, el ítem 2 ("Creo que me esfuerzo mucho para lo poco que consigo") obtuvo el índice más bajo con relación a los demás, siendo este panorama diferente a este estudio, en el cual el ítem 3 fue el más bajo ("Me siento bajo de ánimo, como triste, sin motivo aparente").

En este punto cabe resaltar que es probable que los cambios realizados a losítems 5 , 8 y 9 afectaran las respuestas de los estudiantes, y por ende los indicadores resultantes. Por ejemplo, en la investigación de Ramos et al. (2005), el ítem 8 ("Me siento cansado al final de la jornada de trabajo", en el original) obtuvo un indicador de $.43 \mathrm{y}$ en este estudio, el mismo ítem modificado ("Me siento cansado al final de la jornada de estudio") fue de .591. Del mismo modo con el ítem 9 ("Trabajar pensando en los exámenes me produce estrés", en el original) en el estudio citado fue de .57, y aquí el ítem modificado para la presente investigación enfocado más a los estudios ("Estudiar pensando en los exámenes me produce estrés") fue de .734. Los índices de homogeneidad encontrados por Fontana (2011) resultaron de mayor magnitud que los del presente estudio. A partir de estos descubrimientos podría sugerirse analizar la validez de cada ítem en vista de los hallazgos divergentes con relación a los estudios revisados.

Con relación a la estructura factorial, se corroboran los hallazgos de las investigaciones citadas como antecedentes, en cuanto a la unidimensionalidad del reactivo. El porcentaje de varianza explicada por el factor estudiado (43.361\% de la varianza total del instrumento), es mayor que el presentado por la versión original de la Escala (Ramos et al., 2005), ligeramente inferior a lo presentado por Fontana (2011) e inferior que las reportadas por Gonzáles y Landero (2007b) y Bonilla et al. (2009). Esto quizá se deba a que tanto Gonzáles y Landero (2007b) como Bonilla et al. (2009) usaron un análisis de componentes principales, y no un análisis factorial propiamente dicho, lo cual pudo contribuir a que parte de la varianza explicada por ese método que se reporta se deba al error (Pérez \& Medrano, 2010). Cabe considerar que el valor mínimo requerido es del $20 \%$ de varianza explicada para concluir que hay unidimensionalidad (Carmines \& Zeller, 1979), mas en vista de los hallazgos de otras investigaciones, esta podría optimizarse. Con respecto a las saturaciones factoriales, estas oscilaron entre .517 y .718, lo cual es un indicador de solidez a nivel factorial (Zwick \& Velice, 1986).

Estos hallazgos dan cuenta de una buena estructura interna, ya que las relaciones entre losítemes y los componentes del test conforman el constructo que se quiere medir y sobre el que se basarán las interpretaciones (Elosua, 2003).

Entonces, de acuerdo con Elosua (2003) a través de un número mínimo de factores y mayor varianza explicada se trata de proponer un modelo independiente, por lo cual podemos decir que hay evidencias de dimensionalidad con respecto al cansancio emocional. Si bien es cierto para Messick (1995) el análisis factorial exploratorio es una aproximación débil a la validación del constructo, se optó por este tipo de análisis debido a que en ciencias sociales no es fácil especificar con precisión el valor de las correlaciones con cada factor (Morales-Vallejos, 
2013). Sumado a ello, no se tienen hipótesis previas del comportamiento de los ítems en el contexto de estudiantes universitarios peruanos, considerando que la aplicación del análisis factorial confirmatorio viene guiado por supuestos previos (Ferrando \& AnguianoCarrasco, 2010), lo cual no se halla presente en este instrumento, ya que es la primera vez que se trabaja en nuestro medio.

En lo que respecta a la validez convergente, esta se hace en el marco de la validez de constructo en tanto se busca que aquellos constructos que tienen correlatos a nivel teórico, también los tengan a nivel empírico. En este sentido, se espera una correlación significativa con aquellos criterios externos que son teóricamente relevantes (Westen \& Rosenthal, 2003), en vista de que el objetivo de validación del constructo es la de insertar una medida de un constructo en una red nomológica, esto es, para establecer su relación con otras variables con las cuales debería, en teoría, estar asociado positivamente, negativamente, o sin relación aparente, con los elementos de dicha red (Cronbach \& Meehl, 1955). En tal sentido, las correlaciones encontradas tienen base en la revisión realizada anteriormente, dado que la baja eficacia se relaciona con el cansancio emocional, y hay un correlato presente con ansiedad y depresión, lo cual se corrobora con los hallazgos expuestos.

Por último, con relación a la confiabilidad, fue utilizado el coeficiente Alfa Ordinal dado que las variables no son continuas, aspecto que es de suma importancia cuando se desea utilizar el coeficiente alfa de Cronbach (Elosua \& Zumbo, 2008). El indicador fue de .853 para ese estudio, cuyo valor es adecuado, en vista que para Campo-Arias \& Oviedo (2008) el indicador óptimo de confiabilidad está entre .80 y .90, rango en el cual se hallan también los indicadores de las demás investigaciones revisadas.
Es así que de acuerdo a los hallazgos, la Escala de Cansancio Emocional (ECE) cuenta con propiedades psicométricas adecuadas para seguir con estudios de obtención de evidencias de validez utilizando otras estrategias, apoyando así su uso como instrumento de valoración de la fase inicial del burnout académico, teniendo en consideración que los hallazgos no son concluyentes.

Queda pendiente para futuras investigaciones ampliar la muestra y obtener evidencias de validez predictiva, para dar cuenta del carácter insidioso del síndrome. Asimismo, se recomienda examinar las diferencias de acuerdo al sexo y carrera profesional, toda vez que son aspectos importantes a tener en cuenta.

\section{REFERENCIAS}

Alonso, J. (1995). Motivación y aprendizaje en el aula. Cómo enseñar a pensar. Madrid: Santillana.

Aluja, A. (1997). Burnout profesional en maestros y su relación con indicadores de salud mental. Boletín de Psicología, 55, 47-61.

Bonilla, M., Lira, G., Balcázar, P., Enríquez, J. \& Gurrola, G. (2009). Adaptación de la Escala de Cansancio Emocional en adolescentes mexicanos del nivel superior. Interpsiquis. Recuperado de : http://www.psiquiatria.com/articulos/ ansiedad/estres/39521/

Bresó, E. \& Salanova, M., (2005). Efectos significativos del uso de las creencias de ineficacia como componente del burnout académico en estudiantes universitarios. Jornades de Foment de la Investigació. Recuperado de: www.uji.es/bin/publ/ edicions/jfi8/psi/32.pdf.

Caballero, C., Hederich, C. \& Palacio, J. (2009). El burnout académico: delimitación del síndrome y factores asociados a su 
aparición. Revista Latinoamericana de Psicología, 42(1), 131-146.

Campo-Arias, A. \& Oviedo, H. (2008). Propiedades psicométricas de una escala: la consistencia interna. Revista de Salud Pública, 10(5), 831-839.

Carmines, E. \& Zeller, R. (1979). Reliability and validity assessment. London: Sage.

Costa, P. T. \& McCrae, R. R. (1999). NEOPI-R, Inventario de Personalidad NEO Revisado. NEO-FFI, Inventario NEO reducido de Cinco Factores. Madrid: TEA Ediciones.

Cronbach, L. \& Meehl, P. (1955). Construct validity in psychological tests. Psychological Bulletin, 52, 281-302.

Dominguez, S. (2011). Propiedades Psicométricas del Inventario de Depresión Estado Rasgo en estudiantes universitarios. VIII Congreso Nacional de Profesionales y Estudiantes de Psicología. Facultad de Psicología y Trabajo Social. Universidad Inca Garcilaso de la Vega. Lima, Perú.

Dominguez, S. (2012). Propuesta para el cálculo del Alfa Ordinal y Theta de Armor. Revista de Investigación en Psicología, 15(1), 213-217.

Dominguez, S. Villegas, G., Sotelo, N. \& Sotelo, L. (2012). Revisión Psicométrica del Inventario de Ansiedad EstadoRasgo (IDARE) en una muestra de universitarios de Lima Metropolitana. Revista de Peruana de Psicología y Trabajo Social, 1(1), 35-45.

Dominguez, S., Villegas, G., Yauri, C., Mattos E. \& Ramírez F. (2012). Propiedades psicométricas de una escala de autoeficacia para situaciones académicas en estudiantes universitarios peruanos. Revista de Psicología-Universidad Católica San Pablo, 2(1), 27-39.
Elosua, P. (2003). Sobre la validez de los tests. Psicothema, 15(2), 315-321.

Elosua, P. \& Zumbo, B. (2008). Coeficientes de fiabilidad para escalas de respuesta categórica ordenada. Psicothema, 20(4), 896-901.

Ferrando, P.J. \& Anguiano-Carrasco, C. (2010). El análisis factorial como técnica de investigación en psicología. Papeles del Psicólogo, 31(1), 18-33.

Fontana, S. (2011). Estudio preliminar de las propiedades psicométricas de la escala de desgaste emocional para estudiantes universitarios. Revista Argentina de Ciencias del Comportamiento, 3(2), 4448.

Gil-Monte, P. R. (2005). El síndrome de quemarse por el trabajo (Burnout): una enfermedad laboral en la sociedad del bienestar. Madrid: Pirámide.

Goldberg, D. \& Williams, P. (1996). Cuestionario de Salud General GHQ. Barcelona: Masson.

González, M. \& Landero, R. (2007a). Factor Structure of the Perceived Stress Scale (PSS) in a sample from Mexico. The Spanish Journal of Psychology, 10(1), 201-208.

González, M. \& Landero, R. (2007b). Escala de Cansancio Emocional (ECE) para estudiantes universitarios: propiedades psicométricas en una muestra de México. Anales de Psicología, 23(2), 253-257.

Hair, J. F., Anderson, R. E., Tatham, R. E., \& Black, W.C. (2005). Análise Multivariada de dados. Porto Alegre: Bookman.

Ledesma, R. \& Molina, J.G. (2009). Classical Item and Test Analysis with Graphics: the ViSta-CITA Program. Behavior Research Methods, 41(4), 1161-1168.

León, I. \& Montero, O. (2007). A guide for naming research studies in Psychology. 
International Journal of Clinical and Health Psychology. 7(3), 847-862.

Likert, R. (1932). A Technique for the Measurement of Attitudes. Archives of Psychology, 140, 1-55

Lorenzo-Seva, U. \& Ferrando, P. (2007). FACTOR: A computer program to fit the exploratory factor analysis model. University Rovira y Virgili.

Martínez- Martínez, I. \& Marques Pinto, A. (2005). Burnout en estudiantes universitarios de España y Portugal y su relación con variables académicas. Aletheia, 21, 21-30.

Mas, C. \& Medinas, M. (2007). Motivaciones para el estudio en universitarios. Anales de Psicología, 23 (1), 17-24.

Maslach, C. (1997). Inventario de Burnout de Maslach-MBI. Madrid: TEA Ediciones.

Maslach, C., Schaufeli, W. \& Leiter, M. (2001). Job Burnout. Annual Review Psychology, $52,397-422$.

Messick, S. (1995). Standards of validity and the validity of standards in performance assessment. Educational Measurement: Issues and Practice, 15, 5-12.

Miller, W. G. (2003) OpenStat 4 [programa informático]. Recuperado de: http:// www.statprograms4u.com/OpenStat Features.htm.

Morales-Vallejos, P. (2013) El Análisis Factorial en la construcción e interpretación de tests, escalas y cuestionarios. Madrid: Universidad Pontificia Comillas. Recuperado de: http:// www.upcomillas.es/personal/peter/ investigacion/AnalisisFactorial.pdf.

Neveu, J. (2007). Jailed resources: conservations of resources theory as applied to burnout among prison guards. Journal of Organizational Behavior. 28(1), 21-42.
Palenzuela, D. (1983). Construcción y validación de una escala de autoeficacia percibida específica de situaciones académicas. Análisis y Modificación de Conducta, 9(21), 185-219.

Peñacoba, C. \& Moreno, B. (1999). La escala de estresores universitarios (EEU). Una propuesta para evaluar el estrés en grupos de poblaciones específicas. Ansiedad y Estrés, 5(1), 61-78.

Pérez, E. \& Medrano, L. (2010) Análisis Factorial Exploratorio: Bases Conceptuales y Metodológicas. Revista Argentina de Ciencias del Comportamiento, 2(1), 5866.

Ramos, F., Manga, D. \& Morán, C. (2005). Escala de cansancio emocional (ECE) para estudiantes universitarios: Propiedades psicométricas y asociación. Interpsiquis. Recuperado de: http:// www.psiquiatria.com/articulos / estres/20478/.

Sánchez, M., Castañeiras, C. \& Posada, M. (2011). Autoeficacia en estudiantes de psicologia: Estudio de las relaciones entre autopercepción de competencias $y$ estrategias de afrontamiento. Ponencia presentada en el V Congreso Marplatense de Psicologia.

Spielberger, C.D., Agudelo, D. \& Buela-Casal, G. (2008). Inventario de Depresión Estado/ Rasgo (IDER). Madrid: TEA Ediciones.

Spielberger. C. \& Diaz-Guerrero, R. (2007). IDARE: Inventario de Ansiedad Rasgo Estado. México: El Manual Moderno Timmerman, M. \& Lorenzo-Seva, U. (2011). Dimensionality Assessment of Ordered Polytomous Ítems with Parallel Analysis. Psychological Methods, 16, 209-220.

Vásquez, A., Jimenez, R. \& Vásquez, R. (2004). Escala de Autoestima de Rosenberg: 
fiabilidad y validez en población clínica española. Apuntes de Psicología, 22(2), 247-255.

Westen, D., \& Rosenthal, R. (2003). Quantifying construct validity: Two simple measures. Journal of Personality and Social Psychology, 84(3), 608-618.

Young, F.W. (2003). ViSta "The Visual Statistics System". [programa informático]. Recuperado de: URL: http://forrest. psych.unc.edu/research/index.html, el 16.11.11, 2:00 p.m.

Zwick, W. \& Velicer, W. (1986). Comparison of five rules for determining the number of components to retain. Psychological Bulletin, 99, 432-442. 